\title{
Mucinous Cystic Neoplasms Lined by Abundant Mucinous Epithelium Frequently Involve KRAS Mutations and Malignant Progression
}

\author{
HIDEKI SHIBATA ${ }^{1,2,3}$, NOBUYUKI OHIKE ${ }^{2}$, TOMOKO NOROSE ${ }^{2}$, TOMOHIDE ISOBE ${ }^{2}$, \\ REIKA SUZUKI ${ }^{2}$, HIDEYUKI IMAI $^{2}$, AKIRA SHIOKAWA $^{2}$, TAKESHI AOKI ${ }^{3}$, \\ MASAHIKO MURAKAMI ${ }^{3}$, HIROKI MIZUKAMI ${ }^{4}$, JUN-ICHI TANAKA ${ }^{4}$ and MASAFUMI TAKIMOTO ${ }^{1}$ \\ ${ }^{1}$ Department of Pathology, and ${ }^{3}$ Division of General and Gastroenterological Surgery, \\ Department of Surgery, Showa University Hospital, Tokyo, Japan; \\ Departments of ${ }^{2}$ Pathology, and ${ }^{4}$ Gastroenterological Surgery, \\ Showa University Fujigaoka Hospital, Yokohama, Japan
}

\begin{abstract}
Background: Pancreatic and hepatic mucinous cyst neoplasms (MCNs) have a malignant potential, but indolent MCNs are not uncommon. Materials and Methods: The pathological and genetic characteristics of resected MCNs ( $n=15)$ categorized by the amount of mucin of the lining epithelium were investigated. Results: MCNs were divided into two groups: (i) a rich (r)-MCN group ( $n=6)$, in which more than half of the epithelium was lined by abundant mucinous epithelium; and (ii) a poor (p)-MCN group ( $n=9)$, which consisted of the remaining cases. Three patients in the $r$-MCN group showed invasive carcinoma or high-grade dysplasia, whereas all patients in the p-MCN group showed low-grade dysplasia. Mutations of Kirsten rat sarcoma viral oncogene homolog (KRAS) were more frequent in the $r-M C N$ group (83\%) (p-MCN; 11\%, p<0.05). Conclusion: Mucinous MCNs more frequently have KRAS mutations and higher risk of malignant progression.
\end{abstract}

Mucinous cystic neoplasm (MCN) of the pancreas was first described by Compagno and Oertel in 1978 (1). In the most recent World Health Organization (WHO) Classification of Tumours of the Digestive System (2010) (2), MCN was classified as a distinct entity of cystic neoplasms in both

This Article is freely accessible online.

Correspondence to: Hideki Shibata, Department of Pathology, Showa University Fujigaoka Hospital, 1-30 Fujigaoka, Aoba-ku, Yokohama 227-8501, Kanagawa Japan. Tel: +81 459711151, Fax: +81 459726242, e-mail: hshibata@med.showa-u.ac.jp

Key Words: Mucinous cystic neoplasm, pancreas, liver, mucinous epithelium, KRAS mutation, malignant progression. pancreas and liver, and it has been defined as "a cyst-forming epithelial neoplasm, usually showing no communication with the ductal system, composed of cuboidal to columnar, variably mucin-producing epithelium, and associated with ovarian-type subepithelial stroma" (3-5). MCNs tend to occur most often in middle-aged women and are regarded as pre-malignant lesions with significant potential for invasion and metastasis. Therefore, surgical resection is advocated as the primary treatment modality. However MCNs have a low prevalence of invasive carcinoma: indeed, in a recent large series, invasive carcinoma accounted for $3.9 \%$ to $16 \%$ of lesions in the pancreas $(6,7)$ and $10 \%$ of those in the liver (8). Recently, the term MCN has been challenged by Albores-Saavedra et al. (9, 10), who favor dividing MCNs into two types: "pure MCNs" and "nonmucinous cystadenomas of the pancreas with pancreatobiliary phenotype and ovarian-like stroma". The latter is associated with ovarian-type stroma but is lined by a predominantly nonmucinous and non-dysplastic cuboidal epithelium that probably has no malignant potential. This advocacy may affect the treatment policy and surgical indications for pancreatic and hepatic MCNs. In the present study, we divided pancreatic and hepatic MCNs into mucinous and non-mucinous lesions based on the amount of mucin in the lining epithelium and examined whether or not there was any significant difference in the clinical behavior and biological characteristics between the two.

\section{Materials and Methods}

Fifteen cases of MCN of the pancreas or liver surgically resected between 1994 and 2016 at the Authors' Institutes were examined in the present study. A cystic neoplasm lined by cuboidal to columnar, variably mucin-producing epithelial cells and overlying ovarian-type subepithelial stroma, was regarded as MCN. Based on the findings of a light-microscopic examination of entire or multiple hematoxylin and eosin (H\&E)-stained slides (at least five) including 


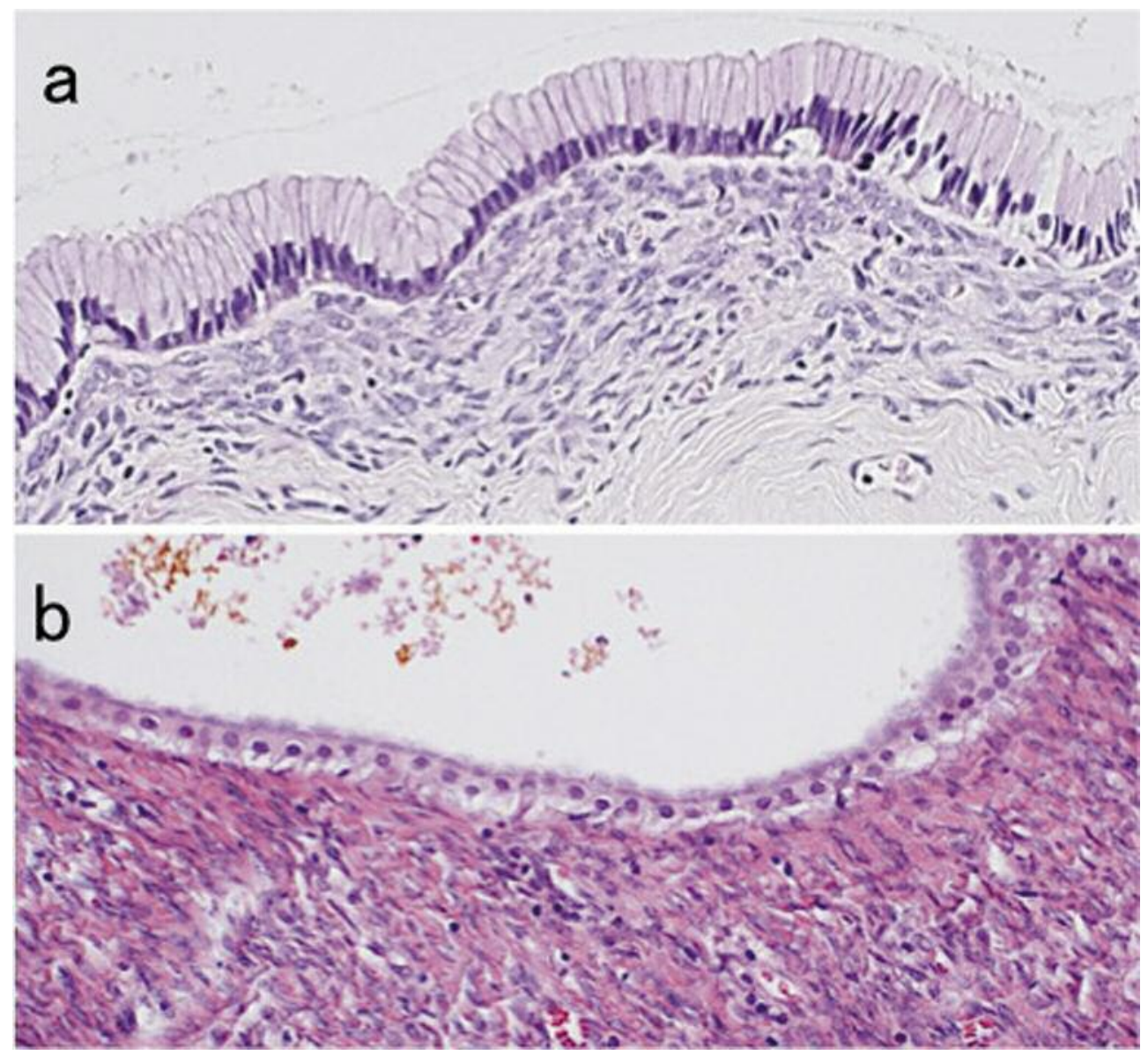

Figure 1. Histology of the lining epithelium of mucinous cystic neoplasms (HE, х400). a: "Mucinous" epithelium; tall columnar epithelium with abundant mucinous cytoplasm. b: "Non-mucinous" epithelium; cuboidal epithelium without obvious mucinous cytoplasm.

the central cut surface of each tumor, all MCNs were divided into two groups: (i) a rich (r)-MCN group, in which arbitrarily more than half of the lining epithelium consisted of tall columnar epithelium with abundant mucinous cytoplasm resembling gastric tall columnar mucinous epithelium (Figure 1a); and (ii) a poor (p)-MCN group, in which more than half of the epithelium was lined by poorly mucinous epithelium characterized by flat to cuboidal or lowcolumnar epithelial cells without obvious mucinous cytoplasm (Figure 1b). The percentage of mucinous epithelium and nonmucinous epithelium was recorded in $5 \%$ increments with the concordance of three observers (H.S., N.O., T. N.).

Several clinicopathological features, including the patient age, sex, location, cyst size, serum tumor markers, histological grade, visible mural nodule, postoperative prognosis, immunohistochemical expression of mucin-1 (MUC1), and Kirsten rat sarcoma viral oncogene homolog (KRAS) mutations were investigated for comparisons between the two groups. The histological grade was classified as low-grade dysplasia, high-grade dysplasia, and invasive carcinoma according to the 2010 WHO classification (2). The duration of postoperative follow-up ranged from 4 to 176 months.
In representative formalin-fixed, paraffin-embedded tissue sections of each case, immunohistochemical staining was performed using the avidin-biotin complex detection method with a BenchMark automated immunostainer (Ventana Medical Systems, Inc., Tucson, AZ, USA). The primary antibodies from Leica Biosystems (Newcastle Ltd, Newcastle, UK) used were against MUC1 (Ma695, 100-fold dilution), MUC2 (Ccp58, 200-fold dilution), MUC5AC (CLH2, 200-fold dilution), MUC6 (CLH5, 50fold dilution), and caudal-type homeobox transcription factor 2 (CDX2) (AMT28, 50-fold dilution). MUC1 was used as a marker for pancreatobiliary-type epithelium, MUC2 and CDX2 for intestinal-type epithelium, and MUC5AC and MUC6 for gastrictype epithelium. Positive staining $>10 \%$ in tumor cells was regarded as significant. For the targeting of the mucinous epithelium of the same tissue sections, an analysis of KRAS mutations was performed by a clinical testing company (SRL, Yokohama, Japan) using the reverse sequence specific oligonucleotide (PCR-RSSO) method.

Statistical analyses were carried out using Fisher's exact or the Mann-Whitney $U$-test, and differences with a value of $p<0.05$ was regarded as significant. 
Table I. Demographic and clinical characteristics of the group of rich mucinous cystic neoplasms $(r-M C N)(n=6)$, in which more than half of the epithelium was lined by abundant mucinous epithelium, and a poor ( $p$ )-MCN group.

\begin{tabular}{|c|c|c|c|c|}
\hline Characteristic & $\begin{array}{l}\text { Total } \\
(n=15)\end{array}$ & $\begin{array}{c}\mathrm{r}-\mathrm{MCN} \\
(\mathrm{n}=6)\end{array}$ & $\begin{array}{c}\mathrm{p}-\mathrm{MCN} \\
(\mathrm{n}=9)\end{array}$ & $p$-Value \\
\hline Age (range), years & $49(24-82)$ & $58(36-82)$ & $43(24-63)$ & 0.088 \\
\hline Sex ratio (Male:female) & $0: 15$ & 0:06 & 0:09 & \\
\hline Size of cyst (range), mm & $61(28-100)$ & $63(45-100)$ & $60(28-100)$ & 0.821 \\
\hline Pancreas/liver, $\mathrm{n}$ & $13 / 2$ & $6 / 0$ & $7 / 2$ & 0.141 \\
\hline CEA (range), $\mathrm{ng} / \mathrm{ml}$ & $1.9(0.3-8.3)$ & $2.5(0.3-8.3)$ & $1.5(0.5-3.4)$ & 0.359 \\
\hline CA19-9 (range), U/ml & $23.6(2-123.7)$ & $25.3(2-123.7)$ & $22.4(2-68.6)$ & 0.886 \\
\hline WHO classification & & & & 0.177 \\
\hline Low-grade dysplasia & 12 & 3 & 9 & \\
\hline High-grade dysplasia & 1 & 1 & 0 & \\
\hline Invasive carcinoma & 2 & 2 & 0 & \\
\hline Mural nodule & 2 & 2 & 0 & 0.188 \\
\hline KRAS mutation & 6 & $5(83 \%)^{*}$ & $1(11 \%)^{* *}$ & 0.011 \\
\hline Recurrence & & 1 & 0 & 0.205 \\
\hline
\end{tabular}

CEA: Carcinoembryonic antigen; CA19-9: carbohydrate antigen 19-9; WHO: World Health Organization. KRAS: Kirsten rat sarcoma viral oncogene homolog. *Two with G12D, one each with G13D, G12A, and G13D; **G12C.

\section{Results}

Demographic and clinicopathological characteristics of all 15 MCN cases. All patients were women. The mean age was 49 years (range $=24-82$ years). Cyst size was $61 \mathrm{~mm}$ (range $=28-100 \mathrm{~mm})$. Primary lesions were mostly pancreatic (13/15). Histologically, all tumors showed convincing ovarian-type stroma, and most cases (12/15) demonstrated low-grade dysplasia, with only two cases of invasive carcinoma (Table I).

Clinicopathological comparisons between the two groups. All MCNs showed a heterogeneous mixture of abundant and poor mucinous epithelium in various proportions: the percentage of poor-mucinous epithelium ranged from $5 \%$ to $90 \%(0-25 \%$ in five cases, $25-50 \%$ in one, $50-75 \%$ in three, $75-100 \%$ in six). According to the arbitrary criterion of $50 \%$ mucinous epithelium, six cases belonged to the r-MCN group and nine to the p-MCN group (Table I). Between the two groups, there were no significant differences in the investigated clinicopathological features of age, cyst size, primary location, and serum tumor marker values; carcinoembryonic antigen and carbohydrate antigen 19-9 values, although the r-MCN group tended to be somewhat older, and have larger cyst size, and higher values of tumor markers than the p-MCN group (Table I). Viscous mucinous content was noted in some r-MCNs (Figure 2a-c), while relatively serous fluid was observed in some p-MCNs (Figure 2d-f), but investigation of the cyst contents was not performed because of insufficient data. Both of the MCNs with invasive carcinoma were primary pancreatic lesions and belonged to the r-MCN group, containing visible mural nodules (Figure 3 ). The invasive component of one case was a tubular carcinoma, while the other was a mixture of tubular and undifferentiated carcinoma. The former involved neighboring organs at surgery, and the patient showed recurrence of liver metastasis 3 months after surgery and has been receiving chemotherapy (still alive after one year). The latter was localized in the pancreas, and no recurrence was found (still alive after 14 years).

Immunohistochemical staining features. There were no significant differences in the staining features of the mucinous epithelium between the two groups (Table II).

KRAS gene test. KRAS mutations were significantly more frequently detected in the r-MCN group (comprising three MCNs with low-grade dysplasia and two with invasive carcinoma, out of a total of six) than in the p-MCN group (one out of nine cases) ( $p=0.011$ ) (Table I). Regarding the types of mutations, of four MCNs with low-grade dysplasia, two had KRAS G12C, one G13C and one G12A, and two MCNs with invasive carcinoma had KRAS G13D and G12D.

\section{Discussion}

Albores-Saavedra et al. reported that among 31 pancreatic MCNs that met the criteria of the 2010 WHO classification, 22 were typical mucinous MCNs lined by abundant mucinous epithelium, six of which were associated with invasive carcinoma (10). Unlike such tumors, the remaining nine neoplasms displayed a predominantly or exclusively 

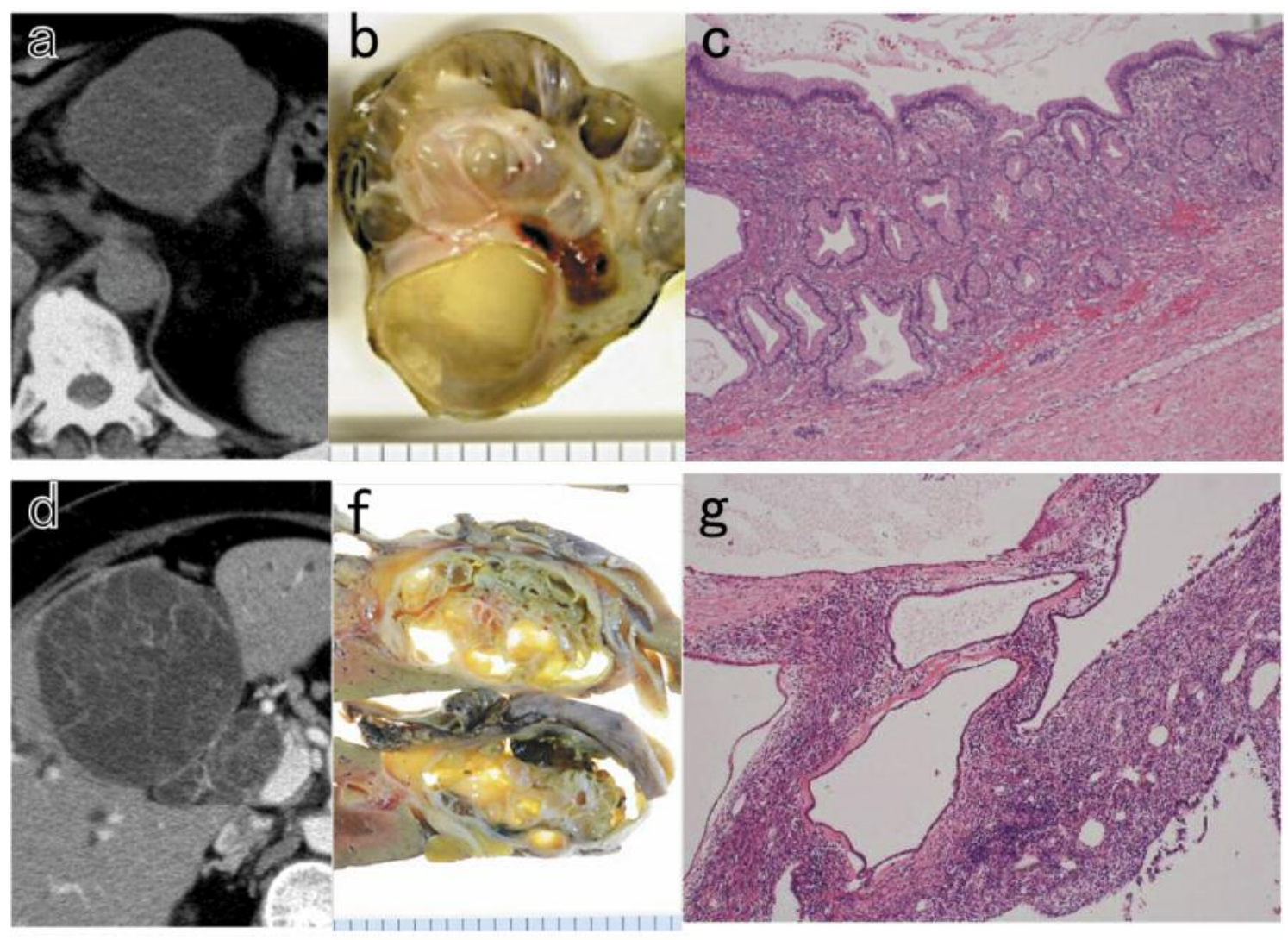

Figure 2. Computed tomography $(a, d)$ and pathological findings (b, $c, f, g)$ of two cases of mucinous cystic neoplasms (MCN) with low-grade dysplasia. a-c: Pancreatic MCN with multilocular cyst $(a, b)$ lined by predominantly abundant mucinous epithelium (c), contained viscous mucin contents (b); classified to the rich MCN group. $d$-g: Hepatic MCN with multilocular cyst ( $d$ and $f$ ), lined by predominantly non-mucinous epithelium $(g)$, contained relatively serous fluid $(f)$ which flowed easily; classified as belonging to the poor MCN group. c, g: HE, x100.
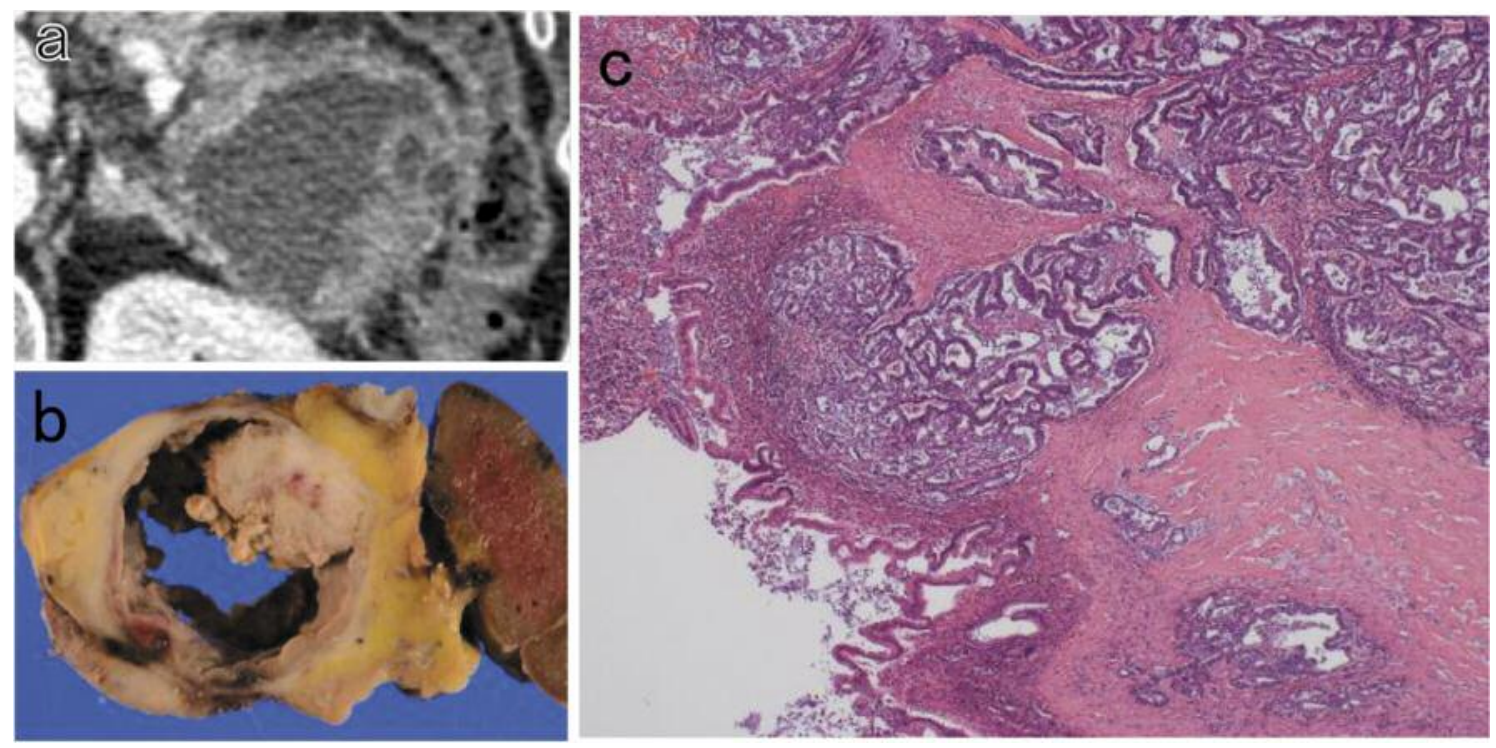

Figure 3. Computed tomography (a) and pathological findings $(b, c)$ of a case of mucinous cystic neoplasms (MCN) with invasive carcinoma. Pancreatic MCN of the rich MCN group, showing visible mural nodules $(a, b)$ of invasive tubular carcinoma $(c)(H E, \times 100)$. 
Table II. Immunohistochemical staining of mucins and caudal-type homeobox transcription factor 2 (CDX2) in the group of rich mucinous cystic neoplasms $(r-M C N)(n=6)$, in which more than half of the epithelium was lined by abundant mucinous epithelium, and a poor ( $p)$-MCN group.

\begin{tabular}{lccc}
\hline & $\mathrm{r}-\mathrm{MCN}(\mathrm{n}=6), \mathrm{n}(\%)$ & $\mathrm{p}-\mathrm{MCN}(\mathrm{n}=9), \mathrm{n}(\%)$ \\
\cline { 2 - 3 } Antigen & Mucinous epithelium & Mucinous epithelium & Non-mucinous epithelium \\
\hline MUC1 & $1(17 \%)$ & $0(0 \%)$ & $4(44 \%)$ \\
MUC5AC & $5(83 \%)$ & $4(44 \%)$ & $3(33 \%)$ \\
MUC6 & $3(50 \%)$ & $3(33 \%)$ & $3(33 \%)$ \\
MUC2 & $0(0 \%)$ & $0(0 \%)$ & $0(0 \%)$ \\
CDX2 & $5(83 \%)$ & $5(56 \%)$ & $5(56 \%)$ \\
\hline
\end{tabular}

Staining $>10 \%$ in tumor cells was considered positive.

non-mucinous single layer of cuboidal or low-columnar nondysplastic epithelial lining similar to the normal ductal epithelium of the pancreas, although they seemed to belong to the family of MCNs, as they were found predominantly in the tail of the pancreas of middle-aged women and exhibited the characteristic ovarian-like stroma that is a hallmark of MCNs. No invasive carcinoma was associated with these neoplasms. Subsequently, Zhelnin et al. also showed that, in a large series of pancreatic and hepatic MCNs, mucinous MCNs with $\leq 50 \%$ non-mucinous epithelium were found in 71 cases, of which eight demonstrated high-grade dysplasia and 14 invasive carcinoma, while MCNs with predominantly (>50\%) nonmucinous epithelium were found in 58 cases, and did not exhibit high-grade dysplasia or invasive carcinoma (11).

Our study, although involving a smaller numbers of cases, reconfirmed the previous finding that MCNs according to the 2010 WHO classification include two types of cystic neoplasms: those with predominantly abundant mucinous cytoplasm (classified as the r-MCN group in the current study) and those with predominantly non-mucinous cytoplasm (classified as the p-MCN group), and only the former were associated with malignant progression (one with high-grade dysplasia and two with invasive carcinoma). Therefore, it seems certain that pancreatic and hepatic MCNs exhibit biologically different characteristics according to the amount of mucin in the lining epithelium, and mucinous MCNs have a higher risk of malignant transformation. These findings also suggest that mucinous MCNs should undoubtedly be resected, whereas non-mucinous MCNs can be observed until resection seems appropriate, because of their indolent behavior.

Accordingly, Albores-Saavedra et al. proposed that such non-mucinous MCNs should be distinguished from typical mucinous MCNs and considered a different entity as "nonmucinous cystadenomas with pancreatobiliary phenotype and ovarian-like stroma" with likely no malignant potential (9, 10). Furthermore, they have applied the same proposal to counterparts in the liver and extrahepatic bile ducts (9). Nonmucinous cystic neoplasms that predominantly consist of a single layer of cuboidal or low-columnar non-dysplastic (benign-looking) cells actually appear to be non-neoplastic or hamartomatous (Figures $1 \mathrm{~b}$ and $2 \mathrm{~g}$ ). However, Zhelnin et $a l$. practice caution in establishing a distinctive entity due to the frequent and indeterminate mixture of mucinous and nonmucinous epithelium (11). They claim that it would be difficult to apply a specific cut-off percentage for differentiating mucinous from non-mucinous cysts given the wide spectrum of amounts of mucinous and non-mucinous epithelium, instead emphasizing that abundant mucinous cytoplasm appears to represent progression to malignancy of MCNs. We experienced similar difficulty as well as subjectivity when establishing the r-MCN and p-MCN groups in the present study. In addition, our data showed no significant clinicopathological or immunohistochemical differences between the two groups. The data on patient age may support the progression theory, as the mean age (58 years) of the r-MCN group was older than that of the $\mathrm{p}-\mathrm{MCN}$ group (43 years). Zhelnin et al. stated in the conclusion of their paper that mucinous epithelium appears to be a prerequisite for carcinomatous transformation, whereas nonmucinous epithelium may simply be a precursor (early phase of the process), since neoplasms with predominantly nonmucinous epithelium were smaller and occurred in younger patients in their series (11).

The KRAS gene belongs to a class of genes known as oncogenes. When mutated, oncogenes have the potential to cause normal cells to become cancerous. Previous studies of KRAS mutations in MCNs have shown that the prevalence of mutations increases with the degree of dysplasia; indeed, $K R A S$ mutations are less frequent in benign and borderline subtypes of MCNs and more common in MCNs with in situ carcinoma or invasive carcinoma $(12,13)$. Jimenez et al. reported KRAS mutations in $20 \%$ of benign, $33 \%$ of borderline, and $89 \%$ of malignant MCNs (13). The current study showed a similar tendency and additionally revealed a 
frequent association between $K R A S$ mutation and mucinous $\mathrm{MCN}$, regardless of the degree of dysplasia. KRAS mutations appear to be strongly associated with mucinous changes in the lining epithelium and are useful as an indicator of the malignant progression phenomenon in MCNs. However, the number of cases examined in the present study was too small to be conclusive, and a larger series is needed to confirm this correlation.

In conclusion, further studies are required to determine whether or not the proposal by Albores-Saavedra et al. that non-mucinous MCNs be considered a different entity is reasonable. However, the recognition of different types of MCNs, such as "MCN, non-mucinous epitheliumpredominant" and "MCN, mucinous epithelium-predominant", would be helpful for characterizing the biology of MCNs and determining treatment policy as recommended by Zhelnin et al. The development and utilization of genetic tests such as KRAS mutation assessments and the application of new techniques and technology, such as confocal laser endomicroscopy, which may allow for the in vivo microscopic imaging of the lining epithelium of cystic neoplasms, may be useful for predicting the risk of progression to malignancy in MCNs. The remaining concern is the validity of examining pancreatic and hepatic MCNs as the same disease. Further accumulation and investigations of cases are required.

\section{Conflicts of Interest}

The Authors have no conflicts of interest to declare.

\section{References}

1 Compagno J and Oertel JE: Mucinous cystic neoplasms of the pancreas with overt and latent malignancy (cystadenocarcinoma and cystadenoma). A clinicopathologic study of 41 cases. Am J Clin Pathol 69: 573-580, 1978.

2 Zamboni G, Fukushima N, Hruban RH and Klöppel G: Mucinous cystic neoplasms of the pancreas. In: World Health Organization Classification of Tumours Pathology and Genetics of Tumours of the Digestive System. Lyon, France: IARC. pp. 300-303, 2010.

3 Thompson LD, Becker RC, Przygodzki RM, Adair CF and Heffess CS: Mucinous cystic neoplasm (mucinous cystadenocarcinoma of low-grade malignant potential) of the pancreas: a clinicopathologic study of 130 cases. Am J Surg Pathol 23: 116, 1999.

4 Ishak KG, Goodman ZD and Stocker JT: Tumors of the liver and intrahepatic bile ducts. In: AFIP Atlas of Tumor Pathology. Rosai J and Sobin LH (eds.). Washington, DC: American Registry of Pathology pp. 51-74, 2001.
5 Suzuki Y, Atomi Y, Sugiyama M, Isaji S, Inui K, Kimura W, Sunamura M, Furukawa T, Yanagisawa A, Ariyama J, Takada T, Watanabe $\mathrm{H}$ and Suda K: Cystic neoplasm of the pancreas: a Japanese multi-institutional study of intraductal papillary mucinous tumor and mucinous cystic tumor. Pancreas 28: 241246, 2004.

6 Yamao K, Yanagisawa A, Takahashi K, Wataru K, Ryuichiro D, Noriyoshi F, Nobuyuki Ohike, Michio S, Takashi H, Bunsei N, Michio H, Yuji K, Kosuke T, Satoshi T, Masanori S, Yoshihiro M, Toshio N, Taketo Y, Keiji H, Hideki A, Minoru T, Naotaka $\mathrm{F}$ and Masao T: Clinicopathological features and prognosis of mucinous cystic neoplasm with ovarian-type stroma: a multiinstitutional study of the Japan Pancreas Society. Pancreas 40: 67-71, 2011.

7 Jang KT, Park SM, Basturk O, Bagci P, Bandyopadhyay S, Stelow EB, Walters DM, Choi DW, Choi SH, Heo JS, Sarmiento JM, Reid MD and Adsay V: Clinicopathologic characteristics of 29 invasive carcinomas arising in 178 pancreatic mucinous cystic neoplasms with ovarian-type stroma: implications for management and prognosis. Am J Surg Pathol 39: 179-187, 2015.

8 Yusuke N, Yuichiro K, Satoshi O, Daigoro T, Rei O, Yasunori N, Kazuhiko K, Naoto G, Shinichiro T and Masaru K: A case of mucinous cystic neoplasm of the liver: a case report. Surg Case Rep 1: 9, 2015.

9 Albores-Saavedra J, Cordova-Ramon JC, Chable-Montero F, Dorantes-Heredia R and Henson DE: Cystadenomas of the liver and extrahepatic bile ducts: Morphologic and immunohistochemical characterization of the biliary and intestinal variants. Ann Diagn Pathol 19: 124-129, 2015.

10 Albores-Saavedra J, Manivel C, Dorantes-Heredia R, ChabléMontero F, Godoy-Valdés C, Chan-Nuñez C and Henson DE: Non-mucinous cystadenomas of the pancreas with pancreatobiliary phenotype and ovarian-like stroma. Am J Clin Pathol 139: 599-604, 2013.

11 Zhelnin K, Xue Y, Quigley B, Reid MD, Choi H, Memis B, Adsay V and Krasinskas AM: Nonmucinous biliary epithelium is a frequent finding and is often the predominant epithelial type in mucinous cystic neoplasms of the pancreas and liver. Am J Surg Pathol 41: 116-120, 2017.

12 Kim SG1, Wu TT, Lee JH, Yun YK, Issa JP, Hamilton SR and Rashid A: Comparison of epigenetic and genetic alterations in mucinous cystic neoplasm and serous microcystic adenoma of pancreas. Mod Pathol 16: 1086-1094, 2003.

13 Jimenez RE, Warshaw AL, Z'graggen K, Hartwig W, Taylor DZ, Compton CC and Fernández-del Castillo C: Sequential accumulation of K-RAS mutations and p53 overexpression in the progression of pancreatic mucinous cystic neoplasms to malignancy. Ann Surg 230: 501-511, 1999.

Received September 8, 2017

Revised October 3, 2017

Accepted October 12, 2017 